\title{
RESEARCH
}

Open Access

\section{miR-34a promotes bone regeneration in irradiated bone defects by enhancing osteoblastic differentiation of mesenchymal stromal cells in rats}

Huan Liu ${ }^{1+}$, Yan Dong ${ }^{1+}$, Xiaoke Feng ${ }^{1+}$, Liya Li ${ }^{2}$, Yang Jiao ${ }^{3}$, Shizhu Bai ${ }^{1}$, Zhihong Feng ${ }^{1}$, Hao Yu ${ }^{1}$, Xuejian Li $^{1}$ and Yimin Zhao ${ }^{1 *}$

\begin{abstract}
Background: Radiation exposure negatively affects the regenerative ability and makes reconstruction of bone defects after tumor section difficult. miR-34a is involved in radiation biology and bone metabolism. The aim of this study was to investigate whether miR-34a could contribute to bone regeneration in irradiated bone defects.

Methods: The expression of miR-34a was analyzed during the osteoblastic differentiation of irradiated BMSCs and bone formation in irradiated bone defects. miR-34a mimics and miR-34a inhibitor were used to upregulate or suppress the expression of miR-34a in BMSCs irradiated with 2 or 4 Gy X-ray radiation. In vitro osteogenesis and subcutaneous osteogenesis were used to assess the effects of miR-34a on the osteogenic ability of radiation-impaired BMSCs. Collagenbased hydrogel containing agomiR-34a or antagomiR-34a were placed into the 3-mm defects of irradiated rat tibias to test the effect of miR-34a on bone defect healing after irradiation.

Results: miR-34a was upregulated in the process of bone formation after irradiation. Transfecting radiation-impaired BMSCs with miR-34a mimics enhanced their osteoblastic differentiation in vitro by targeting NOTCH1. Overexpression of miR-34a enhanced the ectopic bone formation of irradiated BMSCs. In situ delivery of miR-34a promoted bone regeneration in irradiated bone defects.
\end{abstract}

Conclusions: miR-34a promoted the osteoblastic differentiation of BMSCs and enhanced the ectopic bone formation after irradiation. miR-34a promoted bone defect healing in irradiated rat tibias. miR-34a-targeted therapy might be a promising strategy for promoting the reconstruction of bone defects after radiotherapy.

Keywords: miR-34a, Bone marrow mesenchymal stromal cell, Bone regeneration, Osteoblastic differentiation, Radiotherapy

\footnotetext{
* Correspondence: zhaoymdentist@126.com

${ }^{+}$Huan Liu, Yan Dong and Xiaoke Feng contributed equally to this work.

${ }^{1}$ State Key Laboratory of Military Stomatology \& National Clinical Research

Center for Oral Diseases \& Shaanxi Key Laboratory of Stomatology,

Department of Prosthodontics, School of Stomatology, The Fourth Military

Medical University, No. 145 West Changle Road, Xi'an 710032, China

Full list of author information is available at the end of the article
}

C The Author(s). 2019 Open Access This article is distributed under the terms of the Creative Commons Attribution 4.0 International License (http://creativecommons.org/licenses/by/4.0/), which permits unrestricted use, distribution, and reproduction in any medium, provided you give appropriate credit to the original author(s) and the source, provide a link to the Creative Commons license, and indicate if changes were made. The Creative Commons Public Domain Dedication waiver (http://creativecommons.org/publicdomain/zero/1.0/) applies to the data made available in this article, unless otherwise stated. 


\section{Background}

Surgical procedures and radiotherapy are critical components of the multidisciplinary management of bone and soft tissue tumors [1]. The resection of tumors can result in extensive bone defects, and reconstruction of the defect is needed [2]. However, radiotherapy hampers wound healing during bone reconstruction and restoration [3], which lead to higher rates of flap losses [4] and implant failures [5] in patients with radiotherapy.

While destroying potential residual malignant cells, radiotherapy also damages adjacent normal tissues [6]. The hypocellularity, hypovascularity, and hypoxia condition after irradiation inhibits bone growth and healing [7]. In irradiated bone, fewer osteoblast cells and osteocytes are observed, which impedes osteogenesis and bone mineralization [6]. Bone marrow mesenchymal stromal cells (BMSCs) are important source of osteoblasts, but the osteoblastic differentiation of BMSCs is impaired by irradiation $[8,9]$. It is necessary to discover the molecular mechanisms regulating osteogenic differentiation of BMSCs under irradiated conditions.

miR-34a is known as a tumor suppressor that represses the invasion, metastasis, and drug resistance of malignant cells [10]. miR-34a is also involved in tissue development and non-cancerous diseases [11]. It is reported that miR34a plays a role in regulating osteogenic differentiation of stem cells and bone metabolism [12-14]. Irradiation increases the expression of miR-34a in malignant cell lines and normal cells or tissues [15]. However, the function of miR-34a in bone formation after irradiation is unknown.

In this study, we observed that miR-34a was upregulated during the osteoblastic differentiation of irradiated BMSCs and bone formation in irradiated bone defects. Overexpression of miR-34a improved the osteoblastic differentiation of irradiated BMSCs and promoted bone regeneration in bone defects of irradiated rat tibias. miR-34a exerted this effect by repressing NOTCH1. Our findings indicated that miR-34a might be a promising therapeutic target for promoting the reconstruction of the bone defect after malignant tumor resection.

\section{Methods}

\section{Ethics statement}

All animal experiments were approved by the Animal Care Committee of Fourth Military Medical University (Permit Number: kq-022), and all the experimental procedures were conducted following the relevant guidelines and regulations. Sprague-Dawley rats of 2 weeks old or irradiated rats were used for the isolation of BMSCs. Six female nude mice of 6 weeks old were used in the ectopic osteogenesis experiment. Male Sprague-Dawley rats weighing 240-270 g were used for irradiation. Eighteen male Sprague-Dawley rats were used for comparison of miRNA expression and bone regeneration in irradiated and non-irradiated bones. Twelve male Sprague-Dawley rats were used to study the enhancement of miR-34a on bone repair in irradiated bones.

\section{Culture of rat BMSCs and osteoblastic differentiation}

Rat BMSCs were isolated and cultured as described before [16]. Briefly, bone marrow was flushed from tibias and femurs after all soft tissues were removed. The bone marrow suspension was fractionated by density gradient centrifugation (Histopaque-1083, Sigma, MO, USA) for $25 \mathrm{~min}$ at $400 \mathrm{~g}$, and the mononuclear cells were used. Cells were cultured in $\alpha$-minimum essential medium $(\alpha$ MEM) supplemented with 10\% fetal bovine serum (FBS; Sijiqing, Hangzhou, China) and $1 \%$ penicillin and streptomycin. Cells of passage 2 were used in experiments. Sprague-Dawley rats of 2 weeks old were used for the isolation of BMSCs for in vitro test and subcutaneous osteogenesis. BMSCs from irradiated rats were isolated 8 weeks after irradiation.

For osteogenic differentiation, $1 \times 10^{5} /$ well BMSCs were seeded in 12-well plates. Cells were cultured in an osteogenic medium containing $50 \mu \mathrm{g} / \mathrm{ml}$ ascorbic acid, $10 \mathrm{mM}$ $\beta$-glycerophosphate, and $10 \mathrm{nM}$ dexamethasone. Alkaline phosphatase (ALP) staining was tested by BCIP/NBT ALP color development kit (Beyotime, Shanghai, China), and intracellular ALP activity was tested by ALP assay kit (Nanjing Jiancheng Bioengineering Institute, Nanjing, China) according to the manufacturer's protocol after induction for 7 days. Extracellular matrix mineralized nodules were tested by $1 \mathrm{wt} \%$ Alizarin Red S staining (Leagene Biotechnology, Beijing, China) after induction for 21 days. Alizarin Red S staining was further quantitatively analyzed by being dissolved in $10 \%$ cetylpyridinium chloride. The absorbance values were measured at $560 \mathrm{~nm}$. Osteogenesis related mRNA and protein expression were tested after 14 days of osteogenic induction by quantitative real-time reverse transcription-polymerase chain reaction (qRT-PCR) and Western blot.

\section{Irradiation of BMSCs}

When BMSCs reached 95\% confluence, cells were irradiated with $0,2,4$, and $8 \mathrm{~Gy}$ of $\mathrm{X}$-ray radiation delivered at a rate of $1.1 \mathrm{~Gy} /$ minute (RS-2000 XE Biological Irradiator, Rad Source Technologies, GA, USA). The culture medium was replaced by the osteogenic medium after irradiation.

\section{Transfection}

The BMSCs were transfected with miR-34a mimics (50 $\mathrm{nM})$, mimics control (50 nM), miR-34a inhibitor (100 nM), inhibitor control (100 nM), small interfering RNA (siRNA) targeting Notch1 mRNA (50 nM), or negative control (50 nM) (Ruibo, Guangdong, China) using Lipofectamine 2000 after irradiated by 2 or $4 \mathrm{~Gy}$. The medium was replaced by the osteogenic medium $6 \mathrm{~h}$ after transfection. 
RNA extraction and quantitative real-time PCR (qRT-PCR) Total RNA was extracted by TriZol (Invitrogen, CA, USA), and 500 ng total RNA was transcribed into cDNA by a PrimeScript RT reagent kit (TaKaRa, Kyoto, Japan). qRT-PCR was performed with SYBR PremixExTaq ${ }^{\text {Tm II }}$ (TaKaRa) on the CFX96 $6^{\text {tw }}$ Real Time RT-PCR System. Relative expression was calculated by the $\Delta \Delta \mathrm{Ct}$ method, and Gapdh was used for normalization. The primers were synthesized as shown in Table 1. For miRNA quantification, Bulge-loopTM qRT-PCR Primer Sets (one PT primer and a pair of qPCR primers for each set) specific for miR-34a and U6 were designed by Ruibo.

\section{Western blot analysis}

Cells were lysed in RIPA buffer containing a protease inhibitor cocktail (Sigma, MO, USA). Protein concentrations were quantified by the BCA protein assay (Beyotime). Forty micrograms of protein of each sample was loaded on 10\% SDS-PAGE and transferred to the PVDF membranes after separation. The membranes were blocked with $5 \%$ BSA for $2 \mathrm{~h}$ and incubated with primary antibodies for rat COL-1(Protein tech, 14695-1-AP), RUNX2 (Santa Cruz Biotechnology, sc-10758), ALP (Protein tech, 111871-AP), osteocalcin (OCN; Santa Cruz Biotechnology, sc390877), NOTCH1 (Cell Signaling Technology, \#4380), and GAPDH (Abcam, ab8245) overnight at $4{ }^{\circ} \mathrm{C}$. The membranes were incubated for $2 \mathrm{~h}$ with secondary antibodies (Cowin Biotech, China). The protein bands were incubated with a chemiluminescence kit (Amersham Biosciences, USA) and visualized by the imaging system (Tanon 5500, China). The exposure time is $20 \mathrm{~s}$ for GAPDH and 100-200 s for other protein bands depending on the obtained signal intensity. The gray value of the protein bands was quantified by using Image-Pro Plus 6.0 software and normalized to that of GAPDH before comparison.

\section{Subcutaneous osteogenesis model}

For subcutaneous osteogenesis test, $2 \times 10^{5} /$ well BMSCs were seeded in six-well plates and treated with growing medium containing $50 \mu \mathrm{g} / \mathrm{ml}$ vitamin $C$ for 5 days. BMSCs were irradiated with 2 Gy and 4 Gy of X-ray radiation and transfected with miR-34a mimics, mimics control, miR-34a inhibitor, and inhibitor control as described above. The medium was replaced by osteogenic medium $6 \mathrm{~h}$ after

Table 1 Primers used for qRT-PCR

\begin{tabular}{lll}
\hline Gene & Forward primer sequence(5'-3') & Reverse primer sequence(5'-3') \\
\hline Runx2 & 5'-AGA CCA GCA GCA CTC CAT AT-3' & 5'-CTC ATC CAT TCT GCC GCT AGA-3' \\
Alp & 5'-ATG GCT CAC CTG CTT CAC G-3' & 5'-TCA GAA CAG GGT GCG TAG G-3' \\
Ocn & 5'-AGG GCA GTA AGG TGG TGA AT-3' & 5'-GCA TTA ACC AAC ACG GGG TA-3' \\
Col-1 & 5'-GCC TCC CAG AAC ATC ACC TA-3' & 5'-GCA GGG ACT TCT TGA GGT TG-3' \\
Gapdh & 5'-GGCACAGTCAAGGCTGAGAATG-3' & 5'-ATGGTGGTGAAGACGCCAGTA-3' \\
\hline
\end{tabular}

transfection and further cultured for 3 days. The cultured cells along with their deposited extracellular matrix (ECM) were detached as intact sheets from the dishes (BMSC-sheets). Four layers of BMSC-sheets were composited with three layers of bone substitute $(20$ $\mathrm{mg}$ in total, particles $0.25-1 \mathrm{~mm}$, Geistlich) and packaged as a block mass for subcutaneous transplantation as previously described [17]. The implants composed of cell sheets and bone substitute were subcutaneously transplanted into the backs of nude mice $(n=3)$ . Samples were harvested 8 weeks after implantation for histological assay. The implants were fixed by $4 \%$ paraformaldehyde for $48 \mathrm{~h}$ and decalcified with $18 \%$ EDTA for 4 weeks and prepared for H\&E staining.

\section{Irradiation of rats}

Rats were fixed in a perspex jig with their tibias extended laterally. The tibias of rats were subjected to irradiation, and other parts of the body were protected with lead shielding. A single dose of 15 Gy of X-ray radiation was delivered at a rate of $1.1 \mathrm{~Gy} /$ minute (RS-2000 XE Biological Irradiator, Rad Source Technologies).

\section{Rat tibial defect model}

For comparison of miR-34a expression and bone regeneration in irradiated and non-irradiated bones, the right tibia of each rat was irradiated. Bone defect surgeries were conducted 3 weeks after irradiation. A 3-mm tibial defect was generated in both tibias. Rats were sacrificed at 2, 4, and 8 weeks after surgery, and the tibias were harvested. For the test of miR-34a expression, the newly formed bone in the defect area was harvested and ground in liquid nitrogen $(n=3)$. The total RNA was extracted with Trizol reagent (Invitrogen), and miRNA expression was evaluated by qRT-PCR. Newly formed bone was evaluated by MicroCT (Y.Cheetah, Y.XLON, Hamburg, Germany) and H\&E staining $(n=3)$.

To study the enhancement of miR-34a on bone repair in irradiated bone defects, we subjected both tibias of each rat to irradiation. A 3-mm tibial defect was generated in both tibias. Two hundred fifty picomoles of agomir-34a and antagomir-34a, or equal amounts of their respective negative controls $(3 \mu \mathrm{l})$, were dispensed into $15 \mu \mathrm{l} \mathrm{Col-}$ Tgel component $\mathrm{A}$ and $1.5 \mu \mathrm{l}$ component B (Bioruo, Beijing, China), and then were implanted within the defects. At 8 weeks post-implantation, bone regeneration in the defect was evaluated using Micro-CT (Y.Cheetah, Y.XLON) and histological techniques as described below.

\section{Test of micro-CT}

Tibias were harvested and fixed in 4\% paraformaldehyde and scanned by Micro-CT (Y.Cheetah, Y.XLON). Scan settings were rotation of $360^{\circ}$, rotation step of $0.5^{\circ}$, kilovoltage of $80 \mathrm{kV}$, current of $62 \mu \mathrm{A}$, and scanning resolution of 
$18 \mu \mathrm{m}$. VG StudioMAX (Volume Graphics, Heidelberg, Germany) was used for reconstructing 3D images and data analysis. The original bone defect area (L, $2 \mathrm{~mm} ; \phi, 3$ $\mathrm{mm}$ ) was defined as the region of interest (ROI). The percentages of BV/TV were calculated to compare the bone regeneration $(n=5)$.

\section{H\&E staining}

Tibias were decalcified for 4 weeks in $18 \%$ EDTA ( $\mathrm{pH}$ $7.0)$ and embedded in paraffin. Sections ( $5 \mu \mathrm{m}$ thickness) were cut and stained with $\mathrm{H} \& \mathrm{E}$ according to standard protocols.

\section{Sequential fluorescent labelling assay}

To assess bone formation and mineralization in bone defect area, different fluorochromes were injected intramuscularly at a sequence of $30 \mathrm{mg} / \mathrm{kg}$ Alizarin Red S (Sigma), $20 \mathrm{mg} / \mathrm{kg}$ Calcein (Sigma), and $20 \mathrm{mg} / \mathrm{kg}$ Tetracycline Hydrochloride (Sigma) at 3, 5, and 7 weeks after the operation. Tibias were embedded in polymethylmethacrylate (PMMA) and cut into 50- $\mu \mathrm{m}$ sections (LEICA SP1600, Wetzlar, Germany). The undecalcified sections were observed using a confocal laser scanning microscope (OLYMPUS FV1000, Tokyo, Japan), and the area of fluorescent labelling was quantified by Image-Pro Plus 6.0 software $(n=3)$.

\section{Statistical analysis}

Results were displayed as mean \pm standard deviation at least three biological replicates. Differences between two groups were determined by the Student's $t$ test. Differences among groups were analyzed by one-way ANOVA followed by Tukey's post-test. GraphPad Prism7 software was used, and statistical significance was considered when $p<0.05$.

\section{Results}

The expression of miR-34a increased in irradiated BMSCs after osteoblastic differentiation

To verify whether miR-34a is involved in osteoblastic differentiation of radiation-impaired BMSCs, we subjected BMSCs to different doses of X-ray radiation and tested the osteogenesis and the expression of miR-34a. The cultured BMSCs showed typical BMSC characterizations (Additional file 1: Figure S1). X-ray radiation inhibited the osteoblastic differentiation of BMSCs. Both Alp (Fig. 1a) and Alizarin Red S (Fig. 1b) staining were decreased after irradiation. Irradiation caused a reduction in mRNA expression of Alp, Col-1, and Ocn (Fig. 1c). The protein levels of ALP and COL-1 were decreased in irradiated cell. RUNX2 protein levels of the 0 Gy group were higher than the $8 \mathrm{~Gy}$ group. OCN protein levels of the $0 \mathrm{~Gy}$ and $2 \mathrm{~Gy}$ group were higher than $4 \mathrm{~Gy}$ and $8 \mathrm{~Gy}$ group. (Fig. 1d, e). The expression of miR-34a was increased in the $4 \mathrm{~Gy}$ and
8 Gy group $24 \mathrm{~h}$ post-irradiation (0 day after osteogenic induction) compared to the $0 \mathrm{~Gy}$ group. The expression of miR-34a was higher in the 4 Gy and 8 Gy group than 0 Gy group 7 days after osteoblastic differentiation. The expression of miR-34a was higher in all the irradiated groups than 0 Gy group 14 days after osteoblastic differentiation (Fig. 1f).

We also tested the osteogenesis and the miR-34a expression in BMSCs isolated from irradiated (IR-BMSC) and non-irradiated (N-BMSC) rat tibias. IR-BMSC showed lower expression of Runx2, Alp, Col-1, and Ocn than NBMSC 14 days after osteogenic induction (Fig. 1g). The IRBMSC exhibited higher miR-34a level than N-BMSC after osteoblastic differentiation (Fig. 1h).

\section{The expression of miR-34a increased in newly formed bone after irradiation}

Since miR-34a level was higher in the irradiated BMSCs than the non-irradiated BMSCs after osteoblastic differentiation in vitro, we further investigated whether miR-34a was involved in bone formation in vivo after irradiation. Irradiation inhibited bone formation in the defect area (Fig. 2a). The primary callus formation was postponed by irradiation, as the BV/TV of cortical and trabecular bone were lower in the irradiated group than the non-irradiated group at 2 weeks (Fig. 2b, c). Irradiation caused a delay in the osseous closure, as the newly formed cortical bone was less in the irradiated group than in the non-irradiated group at 4 weeks (Fig. 2b). Subsequent remolding was also reduced in the irradiated group, as the BV/TV of trabecular bone was higher in the irradiated group than the nonirradiated group at 4 and 8 weeks (Fig. 2c). The expression level of miR-34a in the newly formed bone was higher in the irradiated group than the non-irradiated group at 2, 4, and 8 weeks after bone defect surgery (Fig. 2d).

\section{miR-34a overexpression enhanced the osteoblastic differentiation of irradiated BMSCs in vitro}

To evaluate the effect of miR-34a on osteoblastic differentiation of irradiated BMSCs, we transfected 2 Gy or 4 Gy irradiated BMSCs with miR-34a mimics, miR-34a inhibitor or their controls. The transfection efficiency was estimated to be $75-90 \%$. Two days after transfection, miR-34a showed a $>150$-fold increase in the miR-34a mimics group and $>38 \%$ reduction in the miR-34a inhibitor group compared with their negative controls. The expression levels remained an 80-fold increase in the miR-34a mimics group and $>30 \%$ reduction in the inhibitor group compared with their negative controls after 14 days of osteogenic induction (Additional file 1: Figure S2).

For 2 Gy irradiated BMSCs, Alp staining and alizarin red staining were increased in the miR-34a mimics group and decreased in the miR-34a inhibitor group compared with their control groups (Fig. 3a). The Alp activity was 


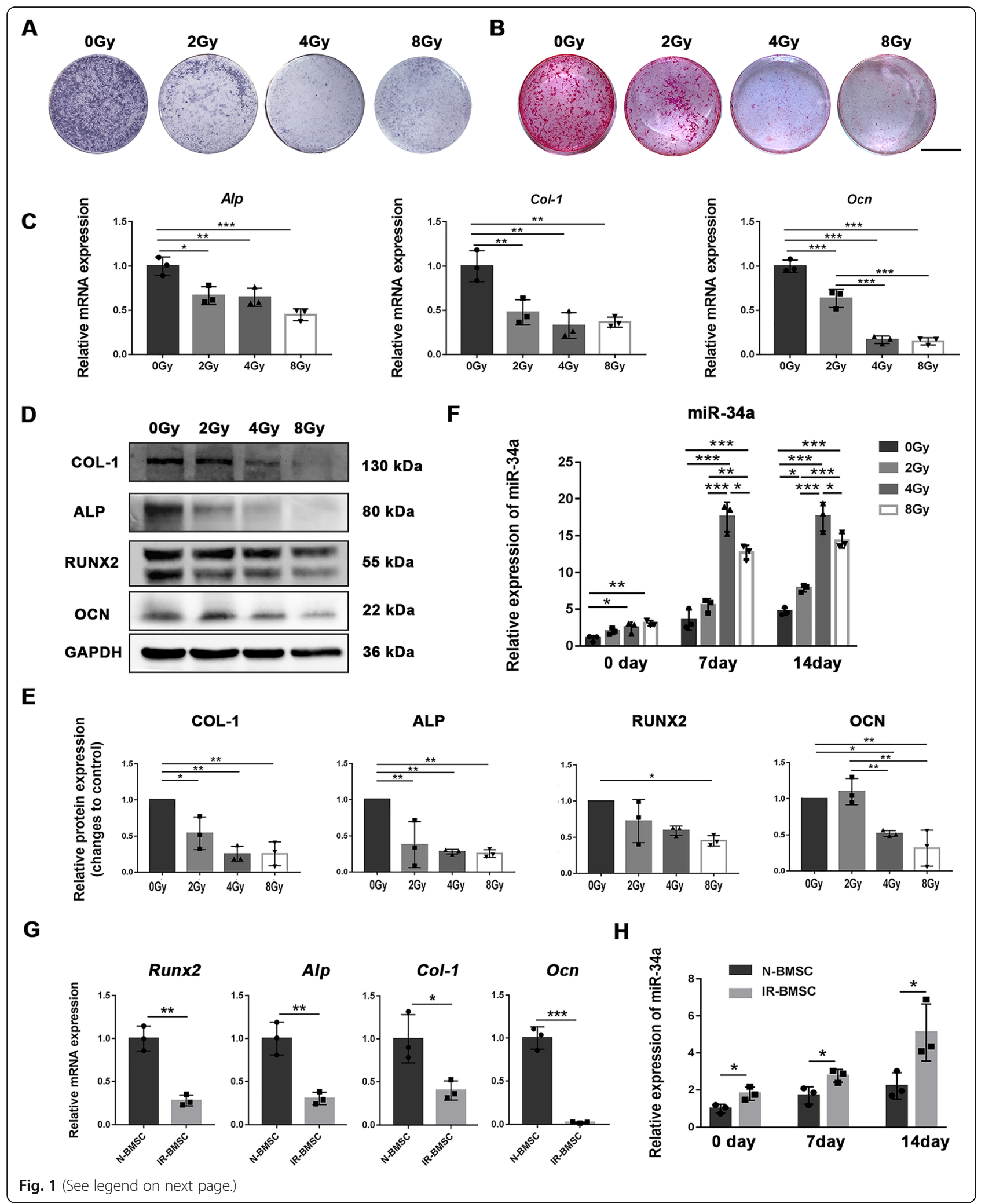


(See figure on previous page.)

Fig. 1 The osteoblastic differentiation and miR-34a expression of irradiated BMSCS. a ALP staining after osteogenic induction for 7 days. b Alizarin red staining after osteogenic induction for 21 days, scale bar $=1 \mathrm{~cm}$. c Gene expression of Runx2, Alp, Col-1, and Ocn after 14 days of osteogenic induction. $\mathbf{d}$ Western blot analysis of RUNX2, ALP, COL-1, OCN, and GAPDH after 14 days of osteogenic induction. e The quantitative analysis of the Western blot results relative to GAPDH (fold to control). f Expression of miR-34a during osteoblastic differentiation. $\mathbf{g}$ Gene expression of Runx2, Alp, Col-1, and Ocn after 14 days of osteogenic induction in the BMSCs isolated from non-irradiated (N-BMSC) and irradiated tibias (IR-BMSC). $\mathbf{h}$ Expression of miR-34a of N-BMSC and IRBMSC during osteogenic differentiation. Data are shown as mean $\pm \mathrm{SD}, n=3 ;{ }^{*} p<0.05$, ${ }^{* *} p<0.01,{ }^{* *} p<0.001$

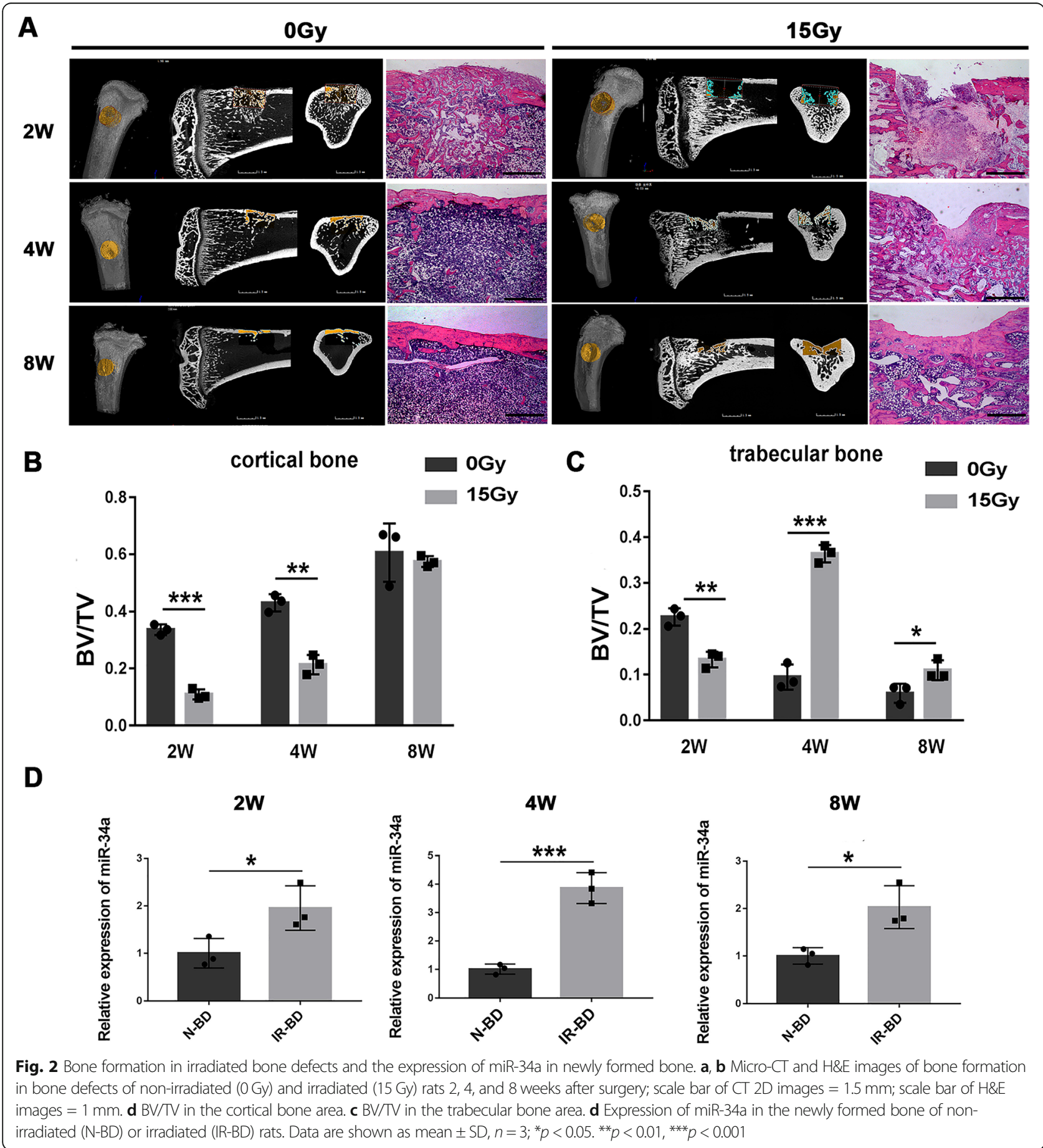


enhanced in the miR-34a mimics group compared with the control group (Fig. 3a). The expression of RUNX2, ALP, COL-1, and OCN at both mRNA and protein levels were enhanced by miR-34a overexpression and reduced by miR34a suppression (Fig. 3b-d). Similar tendency was found in the 4 Gy irradiated BMSCs (Additional file 1: Figure S3).

\section{miR-34a regulated the osteoblastic differentiation of} irradiated BMSCs by repressing NOTCH1

Notch signaling in bone marrow suppresses the osteoblast differentiation of BMSCs [18]. NOTCH1 was previously identified as a direct target of miR-34a in tumor cells [19, 20]. The relationship between miR-34a and NOTCH1 was previously confirmed in human adipose-derived stem cells during osteoblastic differentiation process [14]. To confirm whether miR-34a promotes osteoblastic differentiation of irradiated BMSCs through regulating $\mathrm{NOTCH} 1$, we tested the expression of NOTCH1 in irradiated BMSCs after osteogenic induction. The mRNA expression of Notch1 of 2, 4, and 8 Gy irradiated BMSCs was higher than the nonirradiated BMSCs (Fig. 4a). The protein expression of NOTCH1 was higher in the 4 Gy and 8 Gy group than in the $0 \mathrm{~Gy}$ group. NOTCH1 protein expression was higher in the 8 Gy group than in the 2 Gy group (Fig. $4 \mathrm{c}$ ). NOTCH1 protein expression was downregulated in the miR-34a mimics group and upregulated in the miR-34a inhibitor group compared with their negative controls (Fig. 4d). Notch1 mRNA expression did not show this trend (Fig. 4b).

To further confirm the interaction between NOTCH1 and the osteoblastic differentiation of BMSCs after






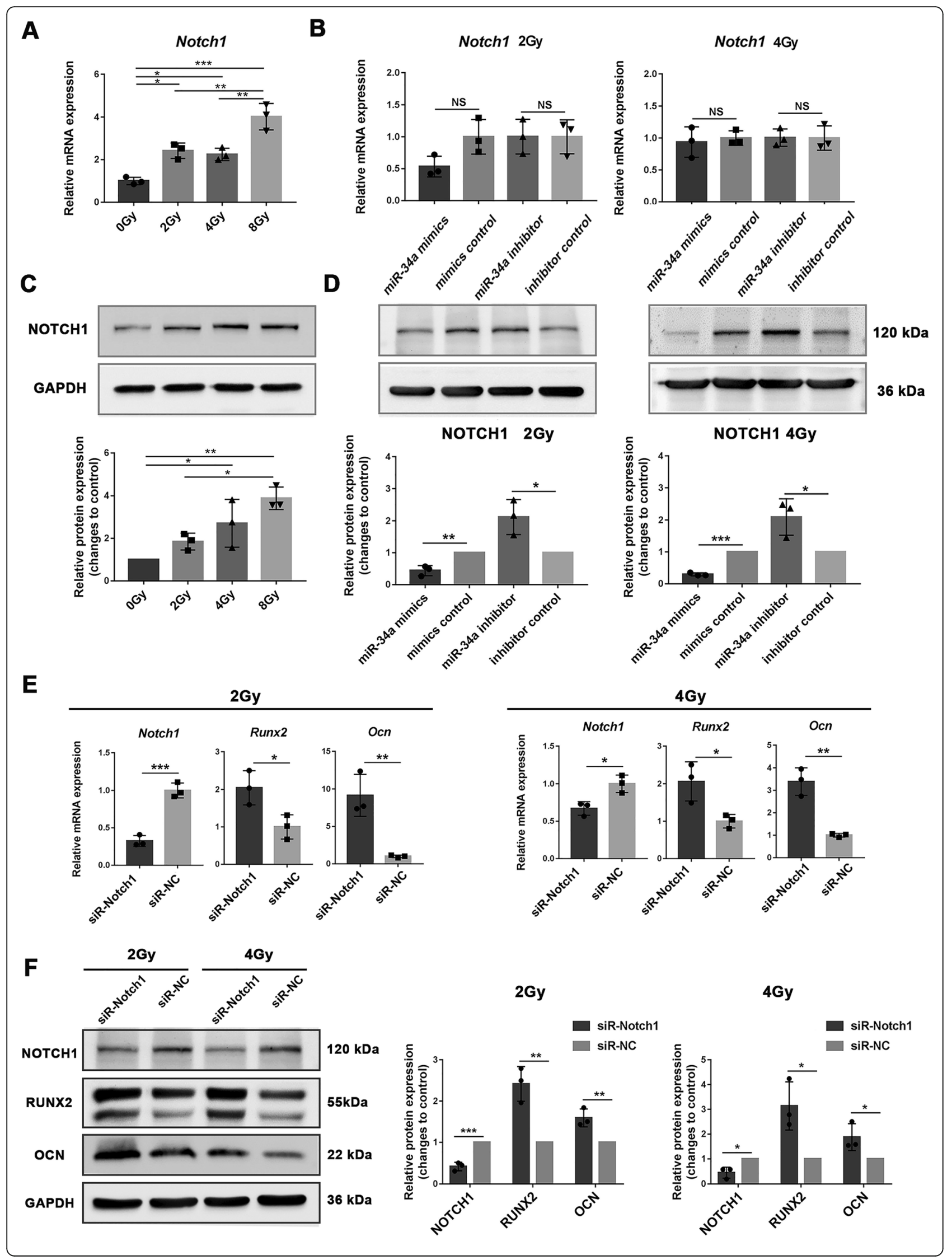


Fig. 4 miR-34a regulated the osteoblastic differentiation of irradiated BMSCs by suppressing NOTCH1. qRT-PCR (a) and Western blot (c) analysis of NOTCH1 in irradiated BMSCs after 14 days of osteogenic induction. qRT-PCR (b) and Western blot (d) analysis of NOTCH1 after 14 days of osteogenic induction for BMSCs irradiated with 2 Gy and 4 Gy. qRT-PCR (e) and Western blot (f) analysis of NOTCH1, RUNX2 and OCN in irradiated BMSCs after NOTCH1 knockdown and 14 days of osteogenic induction. Data are shown as mean \pm SD, $n=3 ;{ }^{*} p<0.05,{ }^{* *} p<0.01,{ }^{* * *} p<0.001$

irradiation, we transfected irradiated BMSCs with siR-Notch. The transfection efficiency was about $80 \%$, and Notch1 mRNA expression was reduced by siRNotch1 2 days after transfection (Additional file 1: Figure S4). After osteogenic induction, the NOTCH1 expression remained decreased in siR-Notch1 groups compared with the control groups (Fig. 4e). Downregulation of NOTCH1 enhanced the mRNA and protein expression of RUNX2 and OCN (Fig. 4e, f). Taken together, these data indicated that miR-34a played a role in regulating osteogenesis under irradiated conditions through NOTCH1.

\section{miR-34a overexpression enhanced the ectopic bone formation of irradiated BMSCs}

To investigate whether miR-34a could improve the ectopic bone formation of irradiated BMSCs, BMSCs irradiated with 2 Gy or 4 Gy were transplanted subcutaneously into nude mice for 8 weeks after transfected with miR-34a mimics, miR-34a inhibitor, or their negative controls. H\&E staining showed that bone formation was enhanced by miR-34a overexpression (Fig. 5a). The percentage of bone area to total area (BA/TA) was used to quantify and compare the amount of newly formed bone. BA/TA was increased by miR-34a overexpression and decreased by miR-34a suppression (Fig. 5b, c).

\section{miR-34a overexpression enhanced bone formation in irradiated bone defects}

To determine whether miR-34a could enhance bone formation in irradiated bone defects, we established a 3-mm tibial bone defect model 3 weeks after irradiation. The collagen-based hydrogel containing agomir-34a, antagomir$34 \mathrm{a}$, or equal amounts of their respective negative controls were placed into the defect sites. New bone formation in the defect was assessed by micro-CT 8 weeks after surgery. More regenerated bone was observed in the agomiR34a group compared with the negative control group (Fig. 6a). BV/TV was higher in the agomiR-34a group than the negative control group (Fig. 6d). The antagomiR-34a group showed less regenerated bone and decreased BV/TV compared with the negative control group (Fig. 6a, d). HE staining (Fig. 6b) confirmed the results of the micro-CT scanning.

Sequential fluorescent labelling was used to measure bone mineralization and deposition during bone defects repair (Fig. 6c), and the labelling area was quantified (Fig. 6e). Alizarin Red S and Calcein labelling area in the agomiR-34a group were larger than in the agomiR control group, while Tetracycline Hydrochloride area in the agomiR-34a group was less than in the agomiR control group. These results indicated that bone formation and subsequent remolding was enhanced by agomiR-34a. Calcein labelling and Tetracycline Hydrochloride area in the antagomiR-34a group were less than in the agomiR control group, indicating that bone defect repair in irradiated tibias was delayed by antagomiR-34a.

\section{Discussion}

The inhibitory effects of radiotherapy on bone formation hinder the reconstruction of bone defects after the resection of bone and soft tissue tumors. In this study, we found a novel strategy based on miRNA to promote bone formation after irradiation. We identified that miR-34a was upregulated during the process of osteogenesis after irradiation. We showed that overexpression of miR-34a promoted the osteoblastic differentiation of irradiated BMSCs. Based on ectopic osteogenesis and tibial defect model, we certified that miR-34a overexpression improved bone regeneration under irradiated conditions.

An increasing number of researches have focused on the osteogenesis and miRNA function, both under the biological and pathological conditions. miR-33-5p is a key factor in the development of osteopenia induced by mechanical unloading, and miR-33-5p-targeted treatment could alleviate the osteopenia [21]. miR-26a was negatively correlated with bone loss in osteoporotic mice, and miR-26a could be used to restore the osteogenic capacity MSCs under osteoporotic circumstance [22]. However, the role of miRNAs in regulating bone formation after irradiation is poorly understood. We found that miR-34a was expressed at a higher level in irradiated BMSCs compared with non-irradiated BMSCs after osteogenic induction. The expression of miR-34a in the regenerated bone within the defect area was also higher in irradiated group than non-irradiated group.

Opinions about the role of miR-34a on osteogenesis are conflicting. Fan reported that overexpression of miR-34a significantly increased the osteogenic differentiation of hASCs [14]. Kang showed that miR-34a diminished the inhibitory effect of dexamethasone on osteoblastic differentiation of mMSC [23]. However, Chen demonstrated that miR-34a is a negative regulator of osteoblast differentiation in hMSC [24]. Dang also showed that inhibition of miR-34a suppressed murine arthritis and bone loss [25]. The inconsistent results might be due to the different characteristics of cells and the distinct regulation of osteogenesis under biological or pathological conditions. We found that miR34a improved the osteogenic differentiation of irradiated 


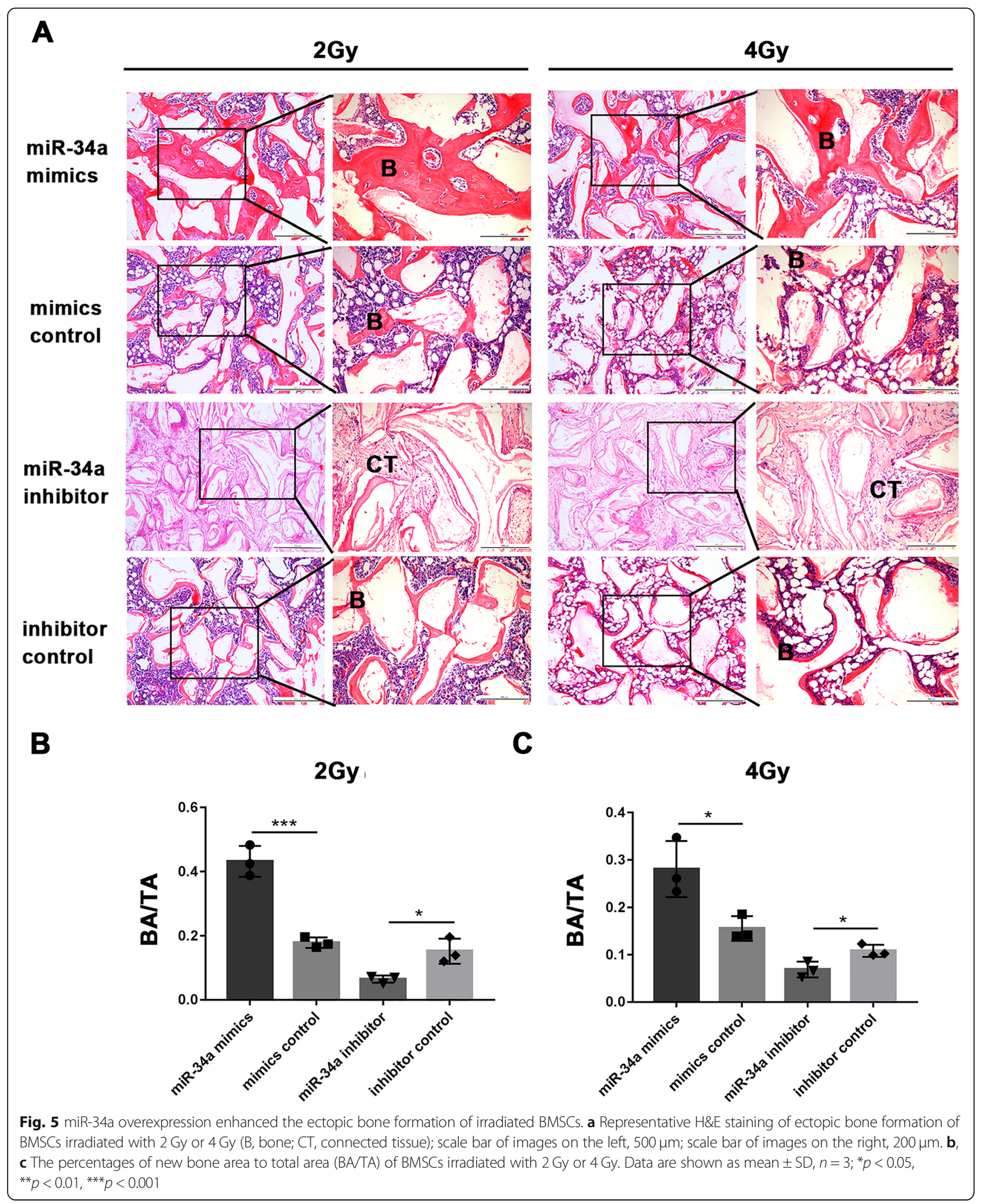

BMSCs in vitro. BMSCs that transfected with miR-34a showed more ectopic bone formation when transplanted into nude mice. miR-34a overexpression also enhanced bone regeneration in irradiated tibial defects. These data suggested that miR-34a could promote osteogenesis under irradiated conditions. 
A
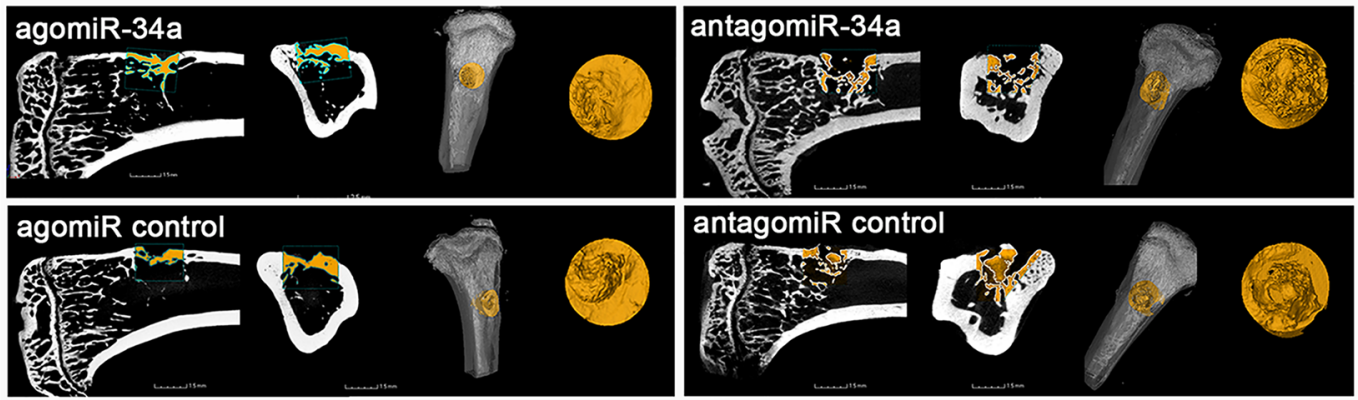

B

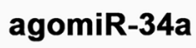

agomiR control

antagomiR-34a

antagomiR control

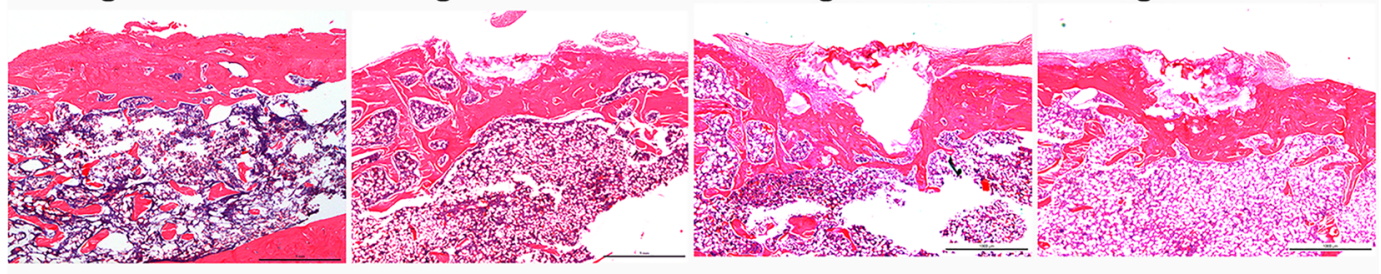

C

agomiR-34a

agomiR control

antagomiR-34a

antagomiR control
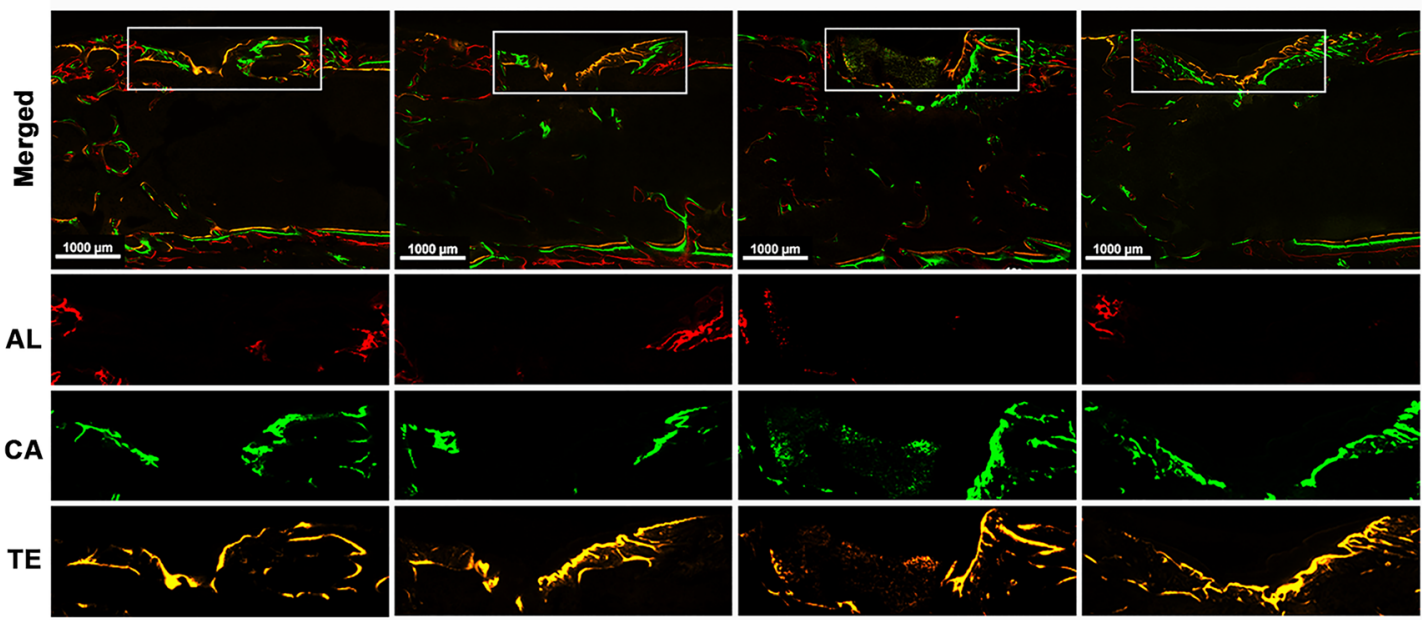

D

E
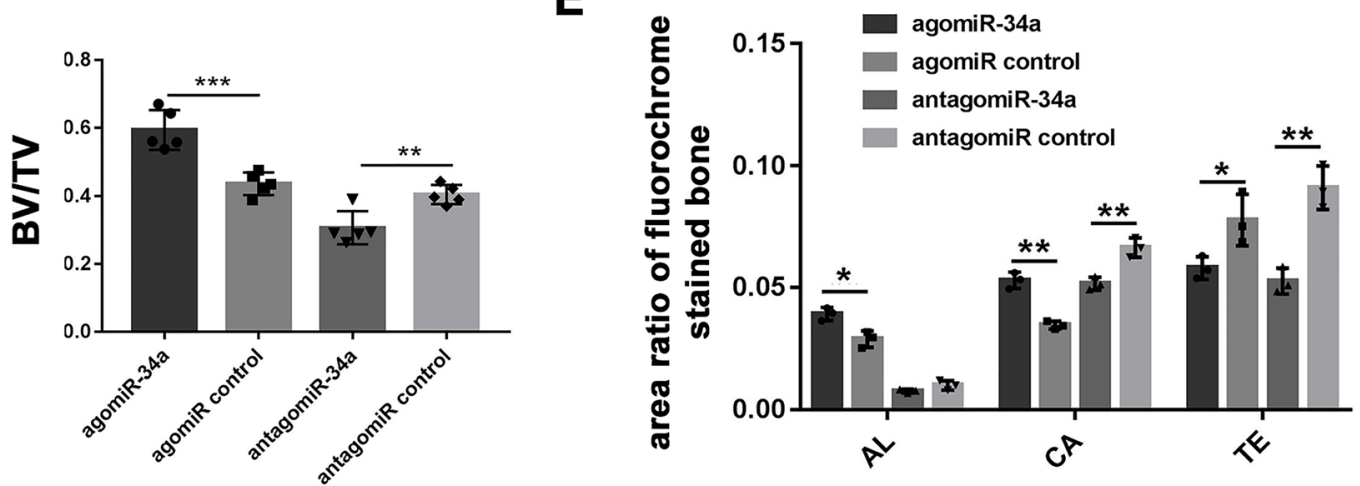

Fig. 6 miR-34a overexpression enhanced bone formation in irradiated bone defects. a 2D and 3D micro-CT images of bone formation in the defect area of irradiated rats at 8 weeks; scale bar of CT 2D images $=1.5 \mathrm{~mm}$. b Representative H\&E staining of new bone formation; scale bar = $1 \mathrm{~mm}$. c Sequential fluorescent labeling of bone formation and mineralization; Alizarin Red S (AL), Calcein (CA), Tetracycline Hydrochloride (TE); scale bar $=1 \mathrm{~mm}$. $\mathbf{d}$ The morphometric analysis of BV/TV for the micro-CT images $(n=5)$. e The quantitative results of sequential fluorescent labeling $(n=3)$. Data are shown as mean \pm SD; ${ }^{*} p<0.05,{ }^{* *} p<0.01,{ }^{* *} p<0.001$ 
Previous studies had verified that NOTCH1 was a direct target for miR-34a by the dual-luciferase reporter assay $[20,26]$. The interaction of miR-34a and NOTCH1 plays a vital role in cell proliferation and apoptosis and is associated with development and disease in some tissues [27]. Notch signaling is also important in bone development and regeneration [28]. miR-34a plays a critical role in bone homeostasis, partly through modulating NOTCH1 $[14,29]$. Our data demonstrated that irradiation enhanced NOTCH1 expression and decreased osteogenesis in BMSCs. Overexpression of miR-34a in irradiated BMSCs led to decreased NOTCH1 protein expression and increased osteogenesis. Furthermore, this study showed that suppression of NOTCH1 enhanced the expression of RUNX2 and OCN in irradiated BMSCs. Our findings suggested that miR-34a improved the osteogenic differentiation of irradiated BMSCs by suppressing NOTCH1. However, we observed that both of the expression of miR-34a and NOTCH1 were increased in irradiated BMSCs compared with nonirradiated BMSCs. As the interaction of miRNAs with their target genes is dynamic and dependent on many factors [30], it is possibly the availability and abundance of miR-34a that made a difference in the interaction between miR-34a and NOTCH1 expression.

Exploring the delivery systems for miRNAs in bone regeneration has gained increased interests. Different miRNA-regulated systems such as systemic injection [31], site-specific injection [32], and scaffold-based delivery were reported. Hydrogels [33], electrospun nanofibers [34], and nanohydroxyapatite scaffold [35] were used for localized delivery of miRNAs. In this research, we used a collagen-based hydrogel for in situ delivery of miR34a. This method upregulated the expression of miR$34 \mathrm{a}$ within the original bone defects for 2 weeks as demonstrated by intracellular uptake of Cy3-labeled agomir (Additional file 1: Figure S5) and qRT-PCR (Additional file 1: Figure S6). We found that miR-34a enhanced the osteogenic differentiation of BMSCs. However, miR34a could also influence the function of other cells involved in bone healing and regeneration. miR-34a could inhibit osteoclast differentiation of osteoclast precursors and reduce bone resorption [12,36]. miR-34a has an antiangiogenic effect on mouse microvascular endothelial cells [37]. A cell-specific miRNA delivery system is needed in the future research to detect the mechanism of miR-34a on bone regeneration after irradiation in vivo.

Reconstruction of the bone defect after malignant tumor resection is difficult as the healing processes are influenced by the tumor and the treatment strategies including surgery, radiotherapy, or chemotherapy [3]. The regenerative medicine strategies of using stem cells, growth factors, and biomaterials to treat bone defects after tumor resection remain controversial for their potential tumor-promoting effects. miR-34a is a tumor suppressor and regarded as a promising therapeutic agent against cancer [38]. Thus, using miR-34a-targeted therapy to enhance bone regeneration in the bone defect after malignant tumor resection may also suppress tumor recurrence.

\section{Conclusion}

In conclusion, this study demonstrated that miR-34a was upregulated in the process of bone formation after irradiation. Overexpression of miR-34a in radiation-impaired BMSCs could promote the osteogenic ability both in vitro and in vivo. In situ delivery of miR-34a promoted bone regeneration in bone defects of irradiated rat tibias. Our findings identified that miR-34a might be a promising therapeutic target for promoting the reconstruction of the bone defect after malignant tumor resection.

\section{Additional file}

Additional file 1: Figure S1. Characterization of BMSCs. Figure S2. The miRNA transfection efficiency and effect. Figure S3. miR-34a overexpression enhanced the osteoblastic differentiation of 4 Gy irradiated BMSCs in vitro. Figure S4. The siRNA transfection efficiency and effect. Figure S5. Distribution of agomiR in the bone defect area. Figure S6. Expression of miR-34a in the newly formed bone after implantation of miRNA delivery hydrogel. (DOCX $2001 \mathrm{~kb})$

\begin{abstract}
Abbreviations
ALP: Alkaline phosphatase; BMSC: Bone marrow mesenchymal stromal cell; COL1: Collagen type I; GAPDH: Glyceraldehyde phosphate dehydrogenase; H\&E: Hematoxylin and eosin; miRNA: microRNA; OCN: Osteocalcin; qRTPCR: Quantitative real-time polymerase chain reaction; RUNX2: Runt-related transcription factor 2; siRNA: Small interfering RNA
\end{abstract}

\section{Acknowledgements}

The authors would like to thank Yan Li and Bei Li for their suggestions on the design of the study.

\section{Funding}

This work was supported by Natural Science Basic Research Plan in Shaanxi Province of China (Program No. 2017JZ026) and Independent Research Project of State Key Laboratory of Military Stomatology (Program No. 2017ZB04).

\section{Availability of data and materials}

All data generated or analyzed during this study are included in this published article.

\section{Authors' contributions}

$H L, Y D$, and XKF performed the experiments and wrote the main manuscript text. $H L$ and $Y D$ designed the experiments. $H Y, X J L$, and $Y J$ helped with the animal test. SZB, ZHF, and LYL interpreted the results. YMZ revised the manuscript. All authors reviewed the manuscript. All authors read and approved the final manuscript.

\section{Ethics approval and consent to participate}

All animal experiments were approved by the Animal Care Committee of Fourth Military Medical University (Permit Number: kq-022), and all the experimental procedures were conducted following the relevant guidelines and regulations. Animals were kept in specific pathogen-free conditions (SPF) at $26^{\circ} \mathrm{C}$ with a $12-\mathrm{h}$ light/dark cycle. They were given a standard pellet rodent diet and water. All surgeries were performed under sodium pentobarbital anesthesia, and all efforts were made to minimize suffering. 


\section{Consent for publication}

Not applicable.

\section{Competing interests}

The authors declare that they have no competing interests.

\section{Publisher's Note}

Springer Nature remains neutral with regard to jurisdictional claims in published maps and institutional affiliations.

\section{Author details}

${ }^{1}$ State Key Laboratory of Military Stomatology \& National Clinical Research Center for Oral Diseases \& Shaanxi Key Laboratory of Stomatology, Department of Prosthodontics, School of Stomatology, The Fourth Military Medical University, No. 145 West Changle Road, Xi'an 710032, China. ${ }^{2}$ Xi'an Institute of Tissue Engineering and Regenerative Medicine, No. 169 West Changle Road, Xi'an 710032, China. ${ }^{3}$ Department of Stomatology, The 7th Medical Center of PLA General Hospital, NO.5, Nanmencang, Dongsishitiao Street, Beijing 100700, China.

Received: 30 October 2018 Revised: 22 April 2019

\section{Accepted: 30 May 2019 Published online: 18 June 2019}

\section{References}

1. Kang SY, Deshpande SS, Zheutlin AR, Donneys A, Rodriguez JJ, Nelson NS, Felice PA, Chepeha DB, Buchman SR. Role of parathyroid hormone in regeneration of irradiated bone in a murine model of mandibular distraction osteogenesis. Head Neck. 2017;39:464.

2. Gharedaghi M, Peivandi MT, Mazloomi M, Shoorin HR, Hasani M, Seyf P, Khazaee F. Evaluation of clinical results and complications of structural allograft reconstruction after bone tumor surgery. Arch Bone Jt Surg. 2016;4:236.

3. Holzapfel BM, Wagner F, Martine LC, Reppenhagen S, Rudert M, Schuetz M, Denham J, Schantz JT, Hutmacher DW. Tissue engineering and regenerative medicine in musculoskeletal oncology. Cancer Metastasis Rev. 2016;35:475.

4. Halle M, Eriksson BO, Docherty SA, Sommar P, Hammarstedt L, Gahm C. Improved head and neck free flap outcome-effects of a treatment protocol adjustment from pre- to postoperative radiotherapy. Plast Reconstr Surg Glob Open. 2017;5:e1253.

5. Smith NA, Santiago JJ, de Faria AD, Dos SD, Pellizzer EP, Goiato MC. Irradiated patients and survival rate of dental implants: a systematic review and meta-analysis. J Prosthet Dent. 2016;116:858.

6. Hu WW, Ward BB, Wang Z, Krebsbach PH. Bone regeneration in defects compromised by radiotherapy. J Dent Res. 2010;89:77.

7. Zheutlin AR, Deshpande SS, Nelson NS, Kang SY, Gallagher KK, Polyatskaya Y, Rodriguez JJ, Donneys A, Ranganathan K, Buchman SR. Bone marrow stem cells assuage radiation-induced damage in a murine model of distraction osteogenesis: a histomorphometric evaluation. Cytotherapy. 2016;18:664.

8. Islam MS, Stemig ME, Takahashi Y, Hui SK. Radiation response of mesenchymal stem cells derived from bone marrow and human pluripotent stem cells. J Radiat Res. 2015;56:269.

9. Wang Y, Zhu G, Wang J, Chen J. Irradiation alters the differentiation potential of bone marrow mesenchymal stem cells. Mol Med Rep. 2016;13:213.

10. Farooqi AA, Tabassum S, Ahmad A. MicroRNA-34a: a versatile regulator of myriads of targets in different cancers. Int J Mol Sci. 2017;18:2089.

11. Rokavec M, Li H, Jiang L, Hermeking $H$. The $p 53 /$ miR-34 axis in development and disease. J Mol Cell Biol. 2014;6:214.

12. Krzeszinski JY, Wei W, Huynh H, Jin Z, Wang X, Chang TC, Xie XJ, He L, Mangala LS, Lopez-Berestein G, Sood AK, Mendell JT, Wan Y. miR-34a blocks osteoporosis and bone metastasis by inhibiting osteoclastogenesis and Tgif2. NATURE. 2014;512:431.

13. Zha X, Sun B, Zhang R, Li C, Yan Z, Chen J. Regulatory effect of microRNA$34 \mathrm{a}$ on osteogenesis and angiogenesis in glucocorticoid-induced osteonecrosis of the femoral head. J Orthop Res. 2018;36:417.

14. Fan C, Jia L, Zheng Y, Jin C, Liu Y, Liu H, Zhou Y. MiR-34a promotes osteogenic differentiation of human adipose-derived stem cells via the RBP2/NOTCH1/CYCLIN D1 coregulatory network. Stem Cell Rep. 2016;7:236.

15. Lacombe J, Zenhausern F. Emergence of miR-34a in radiation therapy. Crit Rev Oncol Hematol. 2017;109:69.
16. Liu H, Zhou W, Ren N, Feng Z, Dong Y, Bai S, Jiao Y, Wang Z, Zhao Y. Cell sheets of co-cultured endothelial progenitor cells and mesenchymal stroma cells promote osseointegration in irradiated rat bone. Sci Rep. 2017;7:3038.

17. Shuai $Y$, Liao L, Su X, Yu Y, Shao B, Jing H, Zhang $X$, Deng $Z$, Jin $Y$. Melatonin treatment improves mesenchymal stem cells therapy by preserving stemness during long-term in vitro expansion. Theranostics. 2016;6:1899

18. Hilton MJ, Tu X, Wu X, Bai S, Zhao H, Kobayashi T, Kronenberg HM, Teitelbaum SL, Ross FP, Kopan R, Long F. Notch signaling maintains bone marrow mesenchymal progenitors by suppressing osteoblast differentiation. Nat Med. 2008;14:306.

19. Zhang C, Mo R, Yin B, Zhou L, Liu Y, Fan J. Tumor suppressor microRNA-34a inhibits cell proliferation by targeting Notch1 in renal cell carcinoma. Oncol Lett. 2014;7:1689.

20. Zhang X, Ai F, Li X, Tian L, Wang X, Shen S, Liu F. MicroRNA-34a suppresses colorectal cancer metastasis by regulating Notch signaling. Oncol Lett. 2017;14:2325.

21. Wang H, Hu Z, Shi F, Dong J, Dang L, Wang Y, Sun Z, Zhou H, Zhang S, Cao $X$, Zhang G. Osteoblast-targeted delivery of miR-33-5p attenuates osteopenia development induced by mechanical unloading in mice. Cell Death Dis. 2018;9:170.

22. Li Y, Fan L, Hu J, Zhang L, Liao L, Liu S, Wu D, Yang P, Shen $L$, Chen J, Jin Y. MiR-26a rescues bone regeneration deficiency of mesenchymal stem cells derived from osteoporotic mice. Mol Ther. 2015;23:1349.

23. Kang H, Chen H, Huang P, Qi J, Qian N, Deng L, Guo L. Glucocorticoids impair bone formation of bone marrow stromal stem cells by reciprocally regulating microRNA-34a-5p. Osteoporos Int. 2016;27:1493.

24. Chen L, Holmstrom K, Qiu W, Ditzel N, Shi K, Hokland L, Kassem M. MicroRNA-34a inhibits osteoblast differentiation and in vivo bone formation of human stromal stem cells. Stem Cells. 2014;32:902.

25. Dang Q, Yang F, Lei H, Liu X, Yan M, Huang H, Fan X, Li Y. Inhibition of microRNA-34a ameliorates murine collagen-induced arthritis. Exp Ther Med. 2017;14:1633.

26. Long YJ, Liu XP, Chen SS, Zong DD, Chen Y, Chen P. miR-34a is involved in CSE-induced apoptosis of human pulmonary microvascular endothelial cells by targeting Notch-1 receptor protein. Respir Res. 2018;19:21.

27. Wang XP, Zhou J, Han $M$, Chen $C B$, Zheng $Y T$, He XS, Yuan XP. MicroRNA-34a regulates liver regeneration and the development of liver cancer in rats by targeting Notch signaling pathway. Oncotarget. 2017; 8:13264.

28. Engin $F$, Yao Z, Yang $T$, Zhou G, Bertin $T$, Jiang $M M$, Chen $Y$, Wang $L$, Zheng $H$, Sutton RE, Boyce BF, Lee B. Dimorphic effects of Notch signaling in bone homeostasis. Nat Med. 2008;14:299.

29. Wan M, Gao B, Sun F, Tang Y, Ye L, Fan Y, Klein OD, Zhou X, Zheng L. microRNA miR-34a regulates cytodifferentiation and targets multisignaling pathways in human dental papilla cells. PLoS One. 2012;7: e50090.

30. O'Brien J, Hayder H, Zayed Y, Peng C. Overview of MicroRNA biogenesis, mechanisms of actions, and circulation. Front Endocrinol (Lausanne). 2018;9:402.

31. Wang X, Guo B, Li Q, Peng J, Yang Z, Wang A, Li D, Hou Z, Lv K, Kan G, Cao $\mathrm{H}$, Wu H, Song J, Pan X, Sun Q, Ling S, Li Y, Zhu M, Zhang P, Peng S, Xie X, Tang T, Hong A, Bian Z, Bai Y, Lu A, Li Y, He F, Zhang G, Li Y. miR-214 targets ATF4 to inhibit bone formation. Nat Med. 2013;19:93.

32. Yu W, Zheng Y, Yang Z, Fei H, Wang Y, Hou X, Sun X, Shen Y. N-AC-I-LeuPEl-mediated miR-34a delivery improves osteogenic differentiation under orthodontic force. Oncotarget. 2017;8:110460.

33. Nguyen MK, Jeon O, Dang PN, Huynh CT, Varghai D, Riazi H, McMillan A, Herberg S, Alsberg E. RNA interfering molecule delivery from in situ forming biodegradable hydrogels for enhancement of bone formation in rat calvarial bone defects. Acta Biomater. 2018;75:105.

34. James EN, Delany AM, Nair LS. Post-transcriptional regulation in osteoblasts using localized delivery of miR-29a inhibitor from nanofibers to enhance extracellular matrix deposition. Acta Biomater. 2014;10:3571.

35. Mencia Cl, Curtin CM, Duffy GP, O'Brien FJ. Harnessing a novel inhibitory role of miR-16 in osteogenesis by human mesenchymal stem cells for advanced scaffold-based bone tissue engineering. Tissue Eng Part A. 2019;25:24.

36. Lopes HB, Ferraz EP, Almeida AL, Florio P, Gimenes R, Rosa AL, Beloti MM. Participation of microRNA-34a and RANKL on bone repair induced by poly (vinylidene-trifluoroethylene)/barium titanate membrane. J Biomater Sci Polym Ed. 2016;27:1369. 
37. Arunachalam G, Lakshmanan AP, Samuel SM, Triggle CR, Ding H. Molecular interplay between microRNA-34a and Sirtuin 1 in hyperglycemia-mediated impaired angiogenesis in endothelial cells: effects of metformin. J Pharmacol Exp Ther. 2016;356:314.

38. Saito Y, Nakaoka T, Saito H. microRNA-34a as a therapeutic agent against human cancer. J Clin Med. 2015:4:1951.

Ready to submit your research? Choose BMC and benefit from:

- fast, convenient online submission

- thorough peer review by experienced researchers in your field

- rapid publication on acceptance

- support for research data, including large and complex data types

- gold Open Access which fosters wider collaboration and increased citations

- maximum visibility for your research: over $100 \mathrm{M}$ website views per year

At BMC, research is always in progress.

Learn more biomedcentral.com/submissions 